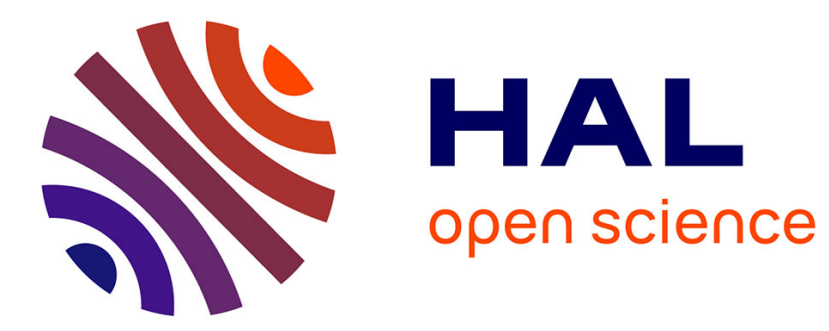

\title{
Probability density function of object contours using regional regularized stochastic watershed
}

Fernando López-Mir, Valery Naranjo, Sandra Morales, Jesus Angulo

\section{To cite this version:}

Fernando López-Mir, Valery Naranjo, Sandra Morales, Jesus Angulo. Probability density function of object contours using regional regularized stochastic watershed. IEEE International Conference on Image Processing (ICIP'2014 ), Oct 2014, Paris, France. pp.4762 - 4766, 10.1109/ICIP.2014.7025965 . hal-01536377

\section{HAL Id: hal-01536377 \\ https://hal-mines-paristech.archives-ouvertes.fr/hal-01536377}

Submitted on 11 Jun 2017

HAL is a multi-disciplinary open access archive for the deposit and dissemination of scientific research documents, whether they are published or not. The documents may come from teaching and research institutions in France or abroad, or from public or private research centers.
L'archive ouverte pluridisciplinaire HAL, est destinée au dépôt et à la diffusion de documents scientifiques de niveau recherche, publiés ou non, émanant des établissements d'enseignement et de recherche français ou étrangers, des laboratoires publics ou privés. 


\section{PROBABILITY DENSITY FUNCTION OF OBJECT CONTOURS USING REGIONAL REGULARIZED STOCHASTIC WATERSHED}

\section{F. López-Mir*, V. Naranjo, S. Morales}

\author{
Instituto Interuniversitario de Investigación \\ en Bioingeniería y Tecnología Orientada \\ al Ser Humano, I3BH/LabHuman. \\ Universitat Politècnica de Valencia. \\ Camino de Vera s/n, 46022 Valencia, Spain
}

\author{
J. Angulo \\ CMM-Centre de Morphologie Mathématique, \\ Mathématiques et Systèmes. \\ MINES Paristech, France. \\ 35, rue Saint-Honoré, 77305 Fontainebleau, \\ France.
}

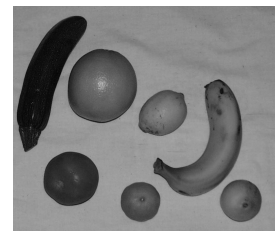

(a)

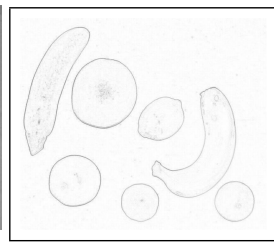

(b)

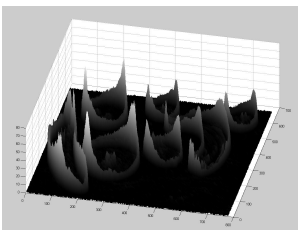

(a) to a region in the output image. To solve this problem, the efforts are focused on the definition of markers to impose new minima in the image, and enhancing the gradient image. The stochastic watershed performs a probability density function (pdf) of the object contours based on a MonteCarlo simulation of random markers. A variation on the method for defining this pdf based on regional regularization of the image is carried out. The objective is to obtain a pdf of the object contours with less noise and better contrast than that produced by the stochastic watershed to use it as a new gradient image for segmentation purposes.

Index Terms - mathematical morphology, segmentation, stochastic watershed, probability density function

\section{INTRODUCTION}

The watershed transform is an image segmentation technique based on mathematical morphology with application in multiple situations [1]. This technique interprets the grayscale image as a topographic surface where the pixel intensity is equal to the altitude, Fig. 1. Each regional minimum of this surface represents a catchment basin, and the idea of the watershed transformation is to flood the surface from these basins. To prevent the merging of two different basins the watershed lines or watersheds are built. These watersheds lines will conform the result of the watershed transform. The standard framework of watershed transform is based on the flooding algorithm, but there are other alternative frameworks: based on

* Thanks to CDTI for funding this work under the project BRAIM (IDI20130020). Corresponding author. Tel.: +34 9638775 18. E-mail address: ferlomir@labhuman.i3bh.es (F. López-Mir).
Fig. 1. Image represented as topographic surface: (a) original image; (b) gradient image; (c) representation as topographic surface of gradient image.

a continuous formulation using topographic distance [2]; the topological watershed based on discrete geometry tools [3]; the graph-based watershed using minimum spanning-tree algorithms [4]; the power watershed algorithm [5]; the viscous watershed [6]; etc.

A segmentation based on the watershed transform is successfully achieved if the minima of the image are located inside of the objects of interest and the edges of these objects are the maxima of the image. For this reason, the gradient image is usually used as input of this transform. The oversegmentation is produced by the great number of minima that appear in the gradient image. The reduction of these minima is the objective of several solutions to improve the watershed transform. One approach is the marker-watershed transform where the minima are imposed by a set of markers introduced artificially in the image gradient [7]. The automatic definition of these markers is not an easy task in some cases but manual markers are user-dependent and the robustness of the watershed transform is limited. The hierarchical watershed paradigms and the waterfall algorithm are based on the strategy of region fusion and the relative weights of the watershed lines that separate the initial basins [8]. Other solutions try to achieve an equivalent image of the object edges that reduces the minima inside of the objects and enhances the boundaries. This is the case of the stochastic watershed [9]. Original 
stochastic watershed is based on Monte Carlo simulations. It was shown in [10] that the corresponding probability density function of contours obtained by stochastic watershed can be calculated using graph algorithms; this latter approach is out of the scope of the paper. More recently, other variants of stochastic watershed has been also proposed [11].

The objective of this paper is to obtain a probability density function of the object edges in a similar way of the original stochastic watershed but taking into account some ideas of the hierarchical and waterfall paradigms in order to achieve an equivalent gradient image less noisy where the regional minima are considerable reduced in comparison to the stochastic watershed or the morphological gradient image. This image will be the input of the watershed algorithm or other variation as the marker-controlled watershed.

The paper is divided as follows. In Section 2, some tools used in this work, the methodology and the mathematical formulation of our approach is explained. Results of several experiments that demonstrate the advantages of this method are presented in Section 3. Finally, in Section 4 the main conclusions are discussed.

\section{METHOD}

\subsection{Tools}

Let $I$ be an image defined as the mapping

$$
I(\mathbf{x}): \mathrm{E} \rightarrow \mathcal{T}
$$

where $\mathbf{x} \in \mathrm{E}$ is the pixel position in the support space of pixels $E$, e.g., for $2 \mathrm{D}$ images $\mathrm{E} \subset \mathbb{N}^{2}$. In the case of discrete images, $\mathcal{T}=\left\{t_{\text {min }}, t_{\text {min }}+1, \ldots, t_{\text {max }}\right\}$ represents the pixel intensity.

The main operators define in mathematical morphology are the dilation $(\delta)$ and the erosion $(\varepsilon)$ and the combination of them produces the opening $(\gamma)$ and the closing $(\varphi)$, the two basic filters in mathematical morphology [12]. The morphological gradient is defined as $\rho(I)=\delta(I)-\varepsilon(I)$. Typically, it is the input image of the watershed algorithm and its variations. With the marker-controlled watershed the minima are imposed by a set of seeds that mark the objects of interest and the background, so the output regions can be controlled according the number of makers defined. The marker-controlled watershed transform is defined as Eq. 2:

$$
W S(\rho(I))_{m(\mathbf{x})}=W S\left(R_{(\rho(I) \wedge m(\mathbf{x}))}^{\varepsilon}(m(\mathbf{x}))\right),
$$

where $m(\mathbf{x})$ is a set of markers:

$$
m(\mathbf{x})= \begin{cases}0, & \text { if } \mathbf{x} \text { belongs to a marker } \\ 255, & \text { otherwise }\end{cases}
$$

and $R_{(\rho \wedge m(\mathbf{x}))}^{\varepsilon}(m(\mathbf{x}))$ is a reconstruction by erosion that imposes the minima of the gradient image, $\rho(I)$, to the pixels set to zero in $m(\mathbf{x})$. Thus, the output regions are limited to the number of connected components of marker image $m(\mathbf{x})$.

In marker-controlled watershed, marker definition is essential to achieve an accurate segmentation. The stochastic watershed uses a different strategy due to the random definition of the markers. This variation is based on an interactive process whose objective is obtain a probability density function of the object contours. The stochastic watershed transform is based on applying $M$ times the marker-controlled watershed with $N$ markers $\left(m_{i}(\mathbf{x})\right)$ that change randomly in each iteration. Using this approach a probability density function can be computed with the Parzen method [13] as Eq. 4.

$$
\operatorname{pdf}(\mathbf{x})=\frac{1}{M} \sum_{i=0}^{M} W S_{i}(\mathbf{x}) * G(\mathbf{x} ; s),
$$

where $W S_{i}(\mathbf{x})=W S(\rho(I))_{m_{i}(\mathbf{x})}$ and $G(\mathbf{x} ; s)$ represents typically a Gaussian kernel of variance $s^{2}$ (in our case, $s=3$ ) and mean $\bar{m}(\bar{m}=0)$, that is defined as:

$$
G(\mathbf{x} ; s)=\frac{1}{2 \pi s^{2}} e^{-\left(\frac{\|\mathbf{x}\|^{2}}{2 s^{2}}\right)} .
$$

Fig. 2 shows some images that produce the algorithm. Fig. 2a and $2 \mathrm{~b}$ are a synthetic image and its respective gradient. This image has internal low gradients that divided the cube in four different regions. This situation tries to simulate when the illumination or contrast is not uniform in an image. Fig. $2 \mathrm{c}$ and $2 \mathrm{~d}$ show two outputs of the marker-controlled watershed with two different set of markers created randomly. In these output images the watershed lines has the same weight and low gradients are enhanced. Fig. 2e is the average of the 30 output images (the watershed lines) of the markercontrolled watersheds carried out following Eq. 4 to obtain the pdf of object contours. In this image, the low gradients that separate the square in four regions are high contrasted while artificial edges that are created in each iteration are reduced by the stochastic procedure. Fig. $2 \mathrm{f}$ is the average of Fig. 2b and 2e proposed in [9] ant it is observable that local gradients intensity are not taken into account and the final segmentation may be worse that in the case of the gradient image is directly used.

\subsection{Regional graylevel-based model}

Stochastic watershed enhances low gradients in the image that can be produced by problems as not uniform illumination or noise. This is justified since the pdf is directly extracted from the gradient image, which contains information about all the local changes in the intensity of the pixels. The stochastic procedure fulfils to reduce irrelevant contours that the watershed itself introduces but it enhances significant contours and also low gradients of the image, Fig. 2. For this reason, in this paper a novel strategy in the way of obtaining the pdf of object contours is presented. In this strategy, irrelevant edges 


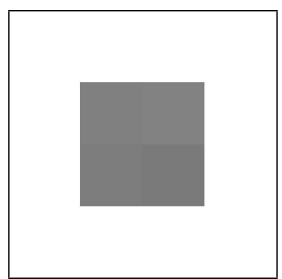

(a)

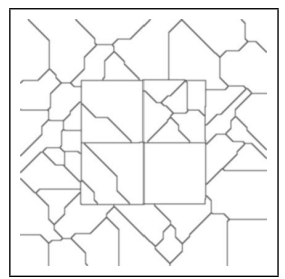

(d)

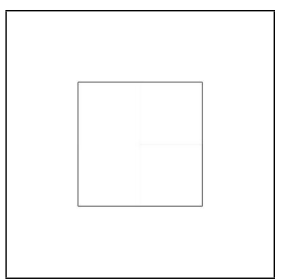

(b)

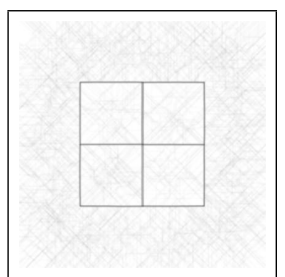

(e)

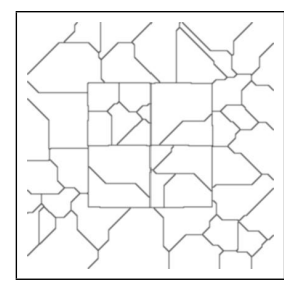

(c)

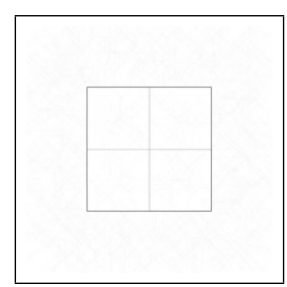

(f)
Fig. 2. Stochastic watershed procedure $(\mathrm{M}=30, \mathrm{~N}=50)$ : (a) original image; (b) gradient image; (c) $j$-th output image of the marker-controlled WS; (d) $i$-th output image of the marker-controlled WS; (e) pdf of stochastic watershed; (f) $($ pdf + gradient $) / 2$.

are also filtered but in each iteration the watershed lines are weighted according to the gradient of the associated region.

The output image of the watershed transform can be also considered by a dual representation as an image partition of the support space $\mathrm{E}$ into $N$ connected classes $\mathcal{C}_{n} \subset \mathrm{E}$, denoted $\Pi(W S(\rho(f)))$ (Eq. 6), where each watershed line which separates two regions (catchment basin) belongs to one of them.

$$
\begin{aligned}
& \Pi(W S(\mathbf{x}))=\left\{\mathcal{C}_{n}\right\}_{1 \leq n \leq N} \\
& \text { with } \bigcup_{1 \leq n \leq N} \mathcal{C}_{n}=\text { E and } \mathcal{C}_{p} \cap \mathcal{C}_{q}=\emptyset, \forall p \neq q
\end{aligned}
$$

The stochastic procedure gives in each iteration an estimation of the gradient image which only measures the local energy of the object contours. In our approach and according to the representation of Eq. 6, each output image of the marker-controlled watershed transform can be represented as an image partition $\Pi$, i.e.,

$$
I(\mathbf{x}) \mapsto \rho(I(\mathbf{x})) \mapsto W S(\mathbf{x}) \mapsto \Pi(W S(\mathbf{x}))
$$

Each $n$-connected class $\mathcal{C}_{n}$ of the partition $\Pi$ is then evaluated with the mean intensity of pixels belonging to this class from the initial image $I$ in order to construct a "mosaic image", denoted by $p(\mathbf{x})$, and formally defined as

$$
p(\mathbf{x})=\left\{\mu_{\mathcal{C}_{n}}(I): \mathbf{x} \in \mathcal{C}_{n}\right\}
$$

where $\mu_{\mathcal{C}_{n}}(I)=1 /\left|\mathcal{C}_{n}\right| \sum_{\mathbf{y} \in \mathcal{C}_{n}} I(\mathbf{y})$ is the average of pixel values of image $I$ in the connected class $\mathcal{C}_{n}$, Fig. $3 \mathrm{~b}$. The morphological gradient of this mean-based simplified model

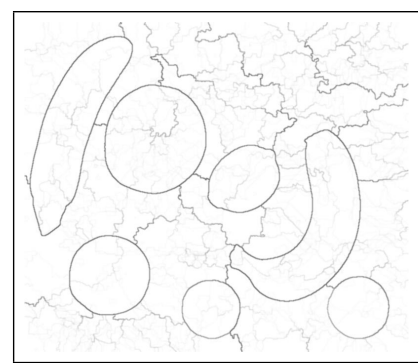

(a)

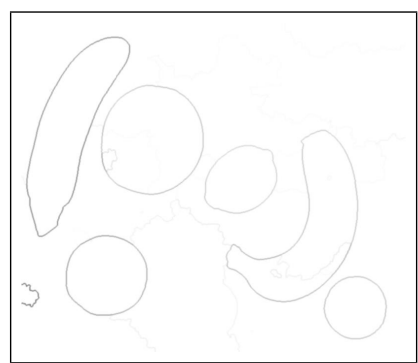

(c)

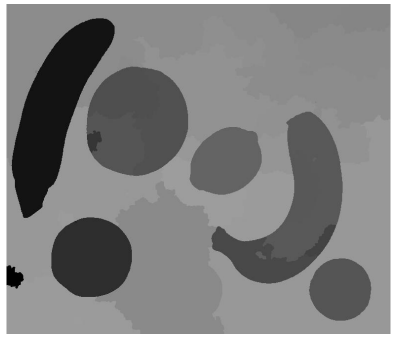

(b)

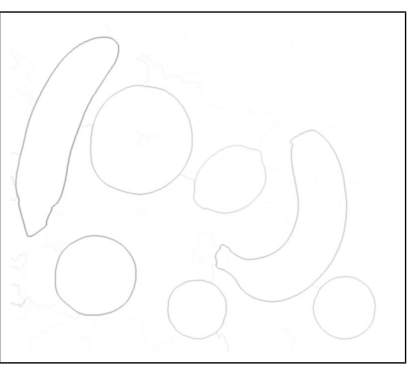

(d)
Fig. 3. Regional graylevel-based model applied in image of Fig.1 ( $\mathrm{M}=30, \mathrm{~N}=50)$ : (a) pdf proposed in [9]; (b) $i$-th mosaic image, $p_{i}(\mathbf{x})$; (c) $\mathcal{E}_{i}(\mathbf{x})$; (d) $\widehat{\operatorname{pdf}}(\mathbf{x})$.

of the image can be interpreted as a regional edgeness energy term, Eq. 9 (Fig. 3c).

$$
\mathcal{E}(\mathbf{x})=\rho(p(\mathbf{x}))
$$

This regional edgeness energy term is introduced in each of the $M$ realizations of the stochastic watershed. That is, by integrating the gradient of the mean-based simplified model of each realization instead of directly the watershed lines, Eq. 10. The objective is to obtain a new probabilistic density function of object contours taking into account local properties of the image, Fig. 3d.

$$
\widetilde{\operatorname{pdf}}(\mathbf{x})=\frac{1}{M} \sum_{i=0}^{M} \mathcal{E}_{i}(\mathbf{x}) * G(\mathbf{x} ; s) .
$$

\section{RESULTS}

Several experiments demonstrate the benefits of the proposed method in comparison with other approaches. The effect of the two parameters $(M, N)$ of the stochastic watershed have been analysed to obtain the number of minima that different methods produced in the pdf of object contours. Fig. 4 shows the image and the gradient used in the next experiments. In this gradient image, the number of regional minima was calculated (the number of output regions of the watershed transform). After, the $N$ parameter was fixed $(N=50)$ and the $M$ parameter was changed between five and one hundred with a 


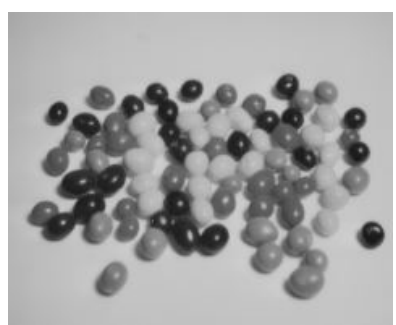

(a)

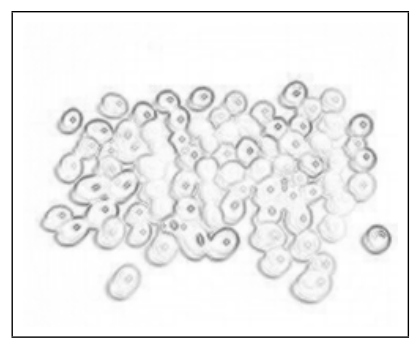

(a)

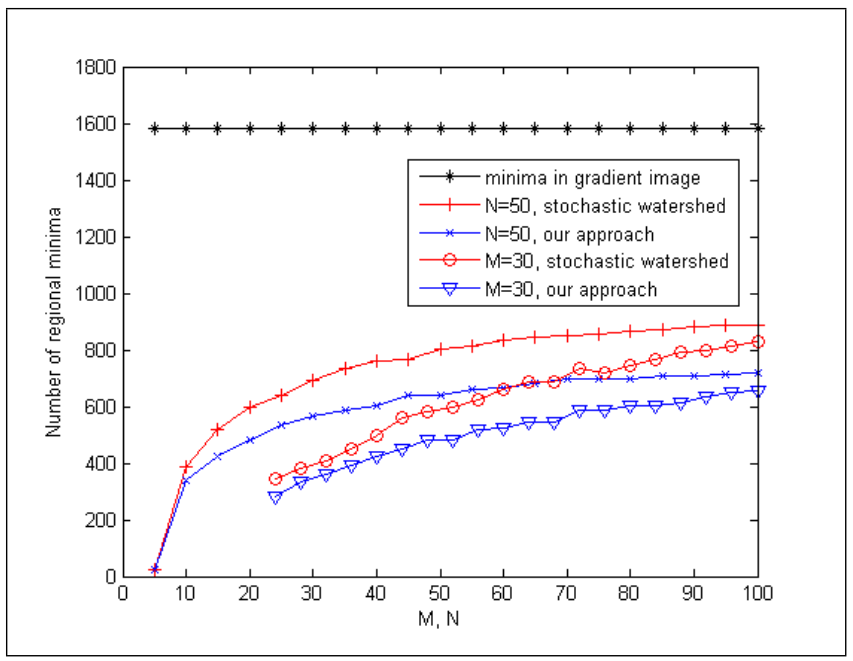

(c)

Fig. 4. (a) original image; (b) gradient image; (c) Number of minima that different methods produce according $M, N$ parameters.

step of five. In each value of $M$, the stochastic watershed was applied five times independently in the gradient image and five pdf of object contours were obtained. Then, The number of minima of each pdf was calculated and the average in each value of $M$ was obtained. This procedure tries to mitigate the random procedure of the whole algorithm. The same steps were carried out with our approach. In other experiment, the same protocol was applied but the $M$ parameter was fixed ( $M=30)$ and the $N$ parameter was changed applying the stochastic watershed and our approach. Fig. 4c summarizes these results, representing the number of minima that our approach and the stochastic watershed produces in relation to the variation of the parameter $M$ (fixing $N$ ) and $N$ (fixing $M$ ) independently.

\section{CONCLUSIONS}

In this paper some of the problems of the watershed transform have been analysed. The problem associated with the number of regional minimum (since each one represents a region in the output image) is the most important to obtain an accurate segmentation with this procedure. This great number

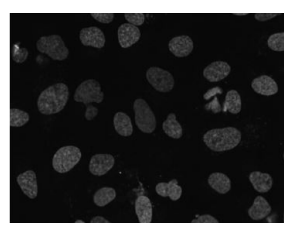

(a)

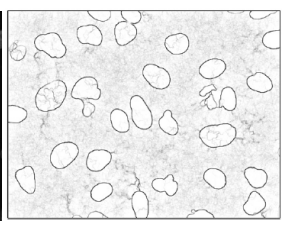

(b)

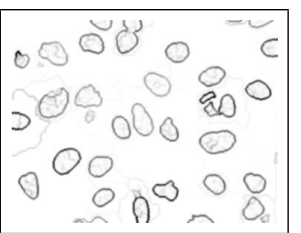

(c)
Fig. 5. (a) image used in [11]; (b) pdf extracted from [11]; (c) pdf of our approach.

of minima is associated with a process of noise, low contrast or non-uniform illumination often introduced at the stage of image capture and it is hardly predictable. To solve this problem, different approaches try to reduce the number of regional minima merging regions or imposing artificial minima in the image. Other approaches seek to improve the image gradient to do it less noisy and/or better enhanced to get a lower number of minima. The stochastic watershed transform creates a probability density function of the object contours based on an iterative process and in the creation of random markers. This process allows to filter the artificial edges that can generate the watershed process itself and generally reduces the number of regions in comparison to the gradient image (Fig.4c) but low gradients sometimes caused by illumination, contrast or noise are enhanced (Fig. 2). To mitigate this problem, some variations in the process of obtaining the pdf of the object contours are proposed in this paper. With this approximation, the number of regional minimum decreases respect to the stochastic watershed (Fig. 4c), since the noise of the pdf is lower than in the original stochastic watershed.

The effects of the parameters $\mathrm{M}, \mathrm{N}$ in our approach are analysed in Fig. 4c. The M parameter can increase, but there is a value where the pdf is stable and if the $M$ value increases results are similar and computational cost increases. The $\mathrm{N}$ parameter has a direct relationship with the number of minima detected in each iteration and the $\widetilde{p d f}$, so the higher value of $\mathrm{N}$, the higher number of regions/minima there is in the $\widetilde{\mathrm{pdf}}$.

In [11] an improvement of the stochastic watershed based on stratified random markers and noise addition is presented. Fig. 5 shows the pdf of our approach and the result presented in [11]. Our approach is less noisy and some objects are better detected than [11]. In all cases under study, the way in which the random markers are generated can be decisive for the final result. In our work, the worst case was taken into account where such markers were generated uniformly and randomly throughout the image in comparison to [11]. In addition, if a priori information was considered the improvement of our approximation can be more significantly than when markers are randomly generated. This would be case of medical images, where much information can be known a priori (organ position, intensity of organs under study, etc.). 


\section{REFERENCES}

[1] Fernand Meyer, "The watershed concept and its use in segmentation : a brief history," CoRR, vol. abs/1202.0216, 2012.

[2] L. Najman and M. Schmitt, "Watershed of a continuous function," Signal Processing, vol. 38, pp. 99-112, 1994.

[3] G. Bertrand, "On topological watersheds," Journal of Mathematical Imaging and Vision, vol. 22, pp. 217-230, 2005.

[4] Fernand Meyer, "Watersheds, waterfalls, on edge or node weighted graphs," CoRR, vol. abs/1204.2837, 2012.

[5] C. Couprie, L. Grady, L. Najman, and H. Talbot, "Power watershed: A unifying graph-based optimization framework," IEEE Transactions on Pattern Analysis and Machine Intelligence, vol. 33, pp. 1384 - 1399, 2011.

[6] Corinne Vachier and Fernand Meyer, "The viscous watershed transform," J. Math. Imaging Vis., vol. 22, pp. 251-267, 2005.

[7] F. Meyer and S. Beucher, "Morphological segmentation," Journal of Visual Communication and Image Representation, vol. 1, no. 1, pp. 21 - 46, 1990.
[8] Serge Beucher, "Watershed, hierarchical segmentation and waterfall algorithm," in Mathematical Morphology and Its Applications to Image Processing, Jean Serra and Pierre Soille, Eds., vol. 2 of Computational Imaging and Vision, pp. 69-76. Springer Netherlands, 1994.

[9] J. Angulo and D. Jeulin, "Stochastic watershed segmentation," in PROC. of the 8th International Symposium on Mathematical Morphology, 2007, pp. 265-276.

[10] Fernand Meyer and Jean Stawiaski, "A stochastic evaluation of the contour strength," in Pattern Recognition, Michael Goesele, Stefan Roth, Arjan Kuijper, Bernt Schiele, and Konrad Schindler, Eds., vol. 6376 of Lecture Notes in Computer Science, pp. 513-522. Springer Berlin Heidelberg, 2010.

[11] Karl B. Bernander, Kenneth Gustavsson, Bettina Selig, Ida-Maria Sintorn, and Cris L. Luengo Hendriks, "Improving the stochastic watershed," Pattern Recognition Letters, vol. 34, no. 9, pp. 993 - 1000, 2013.

[12] Pierre Soille, Morphological Image Analysis, vol. Second edition, Springer, 2002.

[13] R. O. Duda and P. E. Hart, Pattern Classification and Scene Analysis, John Willey \& Sons, New Yotk, 1973. 